\title{
Further analysis of active and passive touch in pattern discrimination
}

\author{
ARTHUR S. SCHWARTZ, ALAN J. PEREY, and ALAN AZULAY \\ Division of Neurobiology, Barrow Neurological Institute of St. Joseph's Hospital and Medical Center, Phoenix, Arizona 85013
}

\begin{abstract}
Discrimination of cookie-cutter patterns by active and passive touch was compared in humans under conditions which attempted to equate the contactual movement of the stimulus over the skin surface in the two modes. We find that the lack of self-initiated movement made no difference in accurate identification of these patterns, contrary to the postulation by Gibson (1962). Further, the results agree with certain neurophysiological findings which suggest that the dorsal columns are critical for the tactile discrimination of spatiotemporal features of stimuli, rather than for mediation of active vs. passive touch per se.
\end{abstract}

Gibson (1962) has distinguished between active and passive touch by characterizing the former as "the impression on the skin brought about by the perceiver himself" and the latter as the impression on the skin brought about "by some outside agency" (p.477). Support for his view that these two modes are nonequivalent is derived from an experiment, mentioned in his theoretical paper above, wherein humans using passive touch (i.e., no movement by the subject's hand while the stimuli are brought into contact with it by the experimenter) were significantly less accurate in identifying cookie-cutter patterns than when they used active touch (i.e., self-initiated exploratory movements by the subject while the patterns were stationary).

Our interest in the distinction between active and passive touch stems from recent findings on the effects of dorsal column lesions in the monkey (Azulay \& Schwartz, 1975; Schwartz, Eidelberg, Marchok, \& Azulay, 1972). Several authors have proposed that this pathway, or the lemniscal system in general, is critical for tactile discriminations involving active touch and is unessential for cutaneous perceptions mediated by passive touch (Schwartz et al., 1972; Schwartzman \& Semmes, 1971; Semmes, 1969; Wall, 1970). The formulation by Wall is the most explicit: he suggests that the dorsal columns are necessary to trigger active exploration of unfamiliar objects during tactile discrimination. Recently, however, we reported that several tactile discriminations in the monkey using active touch were not impaired after dorsal column section (Schwartz et al., 1972). Subsequently, we presented evidence that the dorsal columns were indeed critical if the test objects consisted of patterns which required active exploration for successful discrimination (Azulay \& Schwartz, 1975), and argued that this spinal pathway was necessary for the integration of such spatial patterns rather than for the mediation of active touch discrimination per se. According to our argument, the

This research was supported by USPHS Grant NS 05715 and St. Joseph's Hospital and Medical Center. variable of active touch was irrelevant, and the pattern discriminations would have been equally impaired postoperatively if we had employed passive touch by moving the stimuli over the monkey's immobile fingers. Since Gibson's results are contrary to this expectation, we have modified his experiment to test our hypothesis that no essential difference exists between active and passive tactile discrimination of such patterns, provided that adequate contactual movement is applied across the skin surface.

\section{METHOD}

Ten human volunteers without previous history of neurological disease, of both sexes, ages 19 to 30 years, were used for this study. Each subject sat before a screen with an arm aperture and overhanging baffle so that either arm could be extended through the aperture and rest comfortably during the test. With this arrangement, the subject could not see his hand or the stimulus objects applied by the experimenter.

The forms to be identified were adopted from Gibson (1962) with a few additions (see Figure 1). They were made of stainless
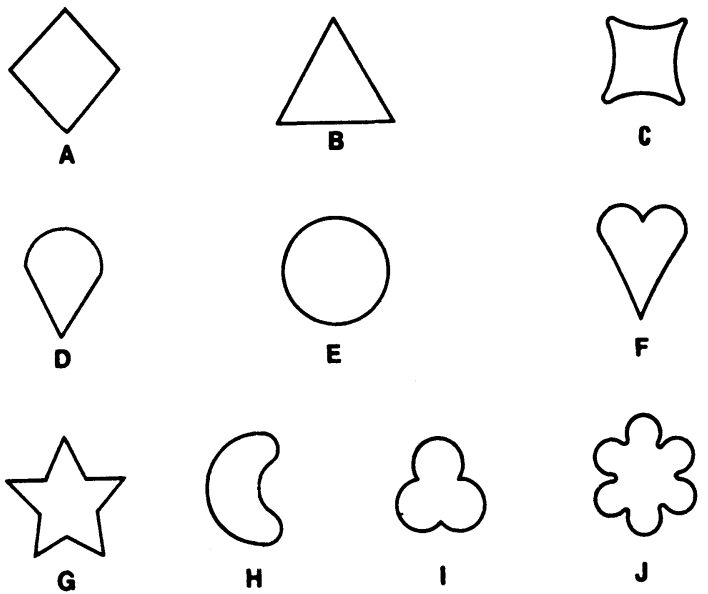

$25 \mathrm{~mm}$

Figure 1. Outline representations of the cookie-cutter patterns. Forms B, C, D, G, H, and $\mathrm{J}$ are copies of those used by Gibson (1962). 
steel, $1.5 \mathrm{~mm}$ thick, and outlined in the style of cookie cutters. In the active mode, the subject was told to trace the contours of each pattern with his index finger until he was sure it corresponded to a figure in a set of numbered drawings equivalent to the set of stimuli; the drawings hung on the screen in front of him, and identification was to be by number. In the passive mode, the subject had to press the back of his hand against a slanted board, with the index finger extended so that the fingernail caught under a thin ledge. This arrangement minimized movements by the subject while the experimenter passed the stimuli against the outstretched finger in approximately the same orientations as during the active mode. The tracing motions by the experimenter were usually in one direction (i.e., clockwise or counterclockwise) and continued until the subject responded. Movement of the objects so as to trace the outlines of the patterns by the experimenter, and using the same finger of the subject as used in the active mode, represent the critical modifications of Gibson's design referred to above. For comparison with the results of Gibson (1962), a "passive static" mode of stimulation was also presented in which the stimuli were mounted on a rotating disk, face down. The subject was instructed to insert his hand into the aperture, palmar surface up, until a brace was encountered which served to position the hand in a standard manner and minimize hand movement. Each stimulus was then dropped onto the center of the palm by a mechanical lever with a force of $800 \mathrm{~g}$, and held there for $2 \mathrm{sec}$. The disk was then raised for $2 \mathrm{sec}$ and the procedure repeated until the subject made a selection.

The patterns were presented twice in each mode in a mixed sequence. The active and passive modes were presented in counterbalanced order, while the passive-static test was presented last. Since preliminary observations indicated improved discrimination with practice, this sequence of tests was expected to enhance the accuracy of the passive-static test. In addition, the active and passive trials were timed from the moment of contact to the verbal report. The data were analyzed by means of the t test, two-tailed.

\section{RESULTS}

Scores for each subject were calculated in terms of percentage correct responses in each mode. The mean frequencies of correct matches in the active and passive modes were $93.5 \%$ and $92.5 \%$, respectively; application of the t test to these data showed that the difference was not significant. By contrast, the passive-static test yielded a mean response of $38.5 \%$ correct, which significantly differed from the active and the passive results $(\mathrm{t}=14.75$ and 13.04 , respectively; $\mathrm{df}=9$; $\mathrm{p}<.001$ in each case) .

As expected, the average time required for a response differed between the active and passive mode $(7.7 \mathrm{sec}$ for the former; $12.9 \mathrm{sec}$ for the latter; $\mathrm{t}=3.47 ; \mathrm{p}<.01$ ). Observation of the tactics used by the subject during the active mode revealed many changes in direction of the finger-tracing movements as the subject sought out the significant features of each contour. In the passive mode, no attempt was made by the experimenter to duplicate this pattern of movements; the only deviations from a continuous uniform passing of the pattern contours over the finger by the experimenter were uncontrolled and unavoidable changes in velocity and pressure.

\section{DISCUSSION}

Our results show that the accuracy of tactile recognition of two-dimensional patterns in the passive mode is not inferior to the active mode when contactual tracing movements are introduced. The scores found here compare closely with Gibson's (1962) reported 95\% accuracy in the active mode. Likewise, the passive-static procedure yielded a coirect identification mean of $38.5 \%$ among our subjects, agreeing with Gibson's result of $29 \%$ and confirming that the latter was significantly less efficient than the active mode or the passive mode with movement. Further comparisons between our scores and those of Gibson must be limited, mainly because the present study employed more patterns than did Gibson, thereby decreasing the probability of making a correct choice by chance.

The finding that subjects required less time in discriminating the patterns during the active mode is not surprising since the subject would be expected to search out the relevant information features of the stimulus, possibly to test ad hoc hypotheses concerning a particular configuration. By following a standard movement routine, we would not expect that the experimenter would present only the key stimulus features as quickly as possible during the passive tests.

Our main concern is with the distinction between active and passive touch, as defined by Gibson. As shown here, perceptual accuracy is not deficient during passive touch if suitable contactual movement between stimulus object and the skin of the sensing organ is arranged by an outside agency. The failure by Gibson to demonstrate the efficacy of passive touch while rotating the cookie patterns in the subject's palm is undoubtedly due to his technique of simply rotating the stimuli in place, as well as using the palm instead of the more sensitive fingers, as he did during his active-mode tests. We do not mean to suggest that active and passive touch, even in the presence of a moving object, are identical perceptual modes in all respects, or that self-initiated movements are not more successful at obtaining information in the tactile natural world than immobile sense organs. Gibson (1962) has clearly pointed out some differences in subjective perceptual experience produced by active vs. passive touch.

The finding here that active and passive pattern discrimination were essentially equivalent modes, in conjunction with other data, are interpreted as indirect support for our concept regarding the tactile role of the dorsal columns. First, section of this pathway does not impair discrimination of various tactile qualities (e.g., roughness, hardness, etc.) in the active mode in monkeys (Schwartz et al., 1972), indicating that the dorsal columns are not critical for active touch per se. However, deficient active touch discrimination can be demonstrated postoperatively if one uses test objects (e.g., patterns) which emphasize the need for exploration of the stimuli-i.e., if contactual movement is required for discrimination (Azulay \& Schwartz, 1975). Second, certain spatiotemporal discriminations in the passive mode are severely impaired after dorsal column lesions (Vierck, 1973, 1974; Wartenberg, 1939). Since the two modes seem equally capable of discriminating patterns such as those for which the dorsal columns appear critical (Azulay \& Schwartz, 1975), we propose that the tactile role of this system is specifically exemplified by tasks requiring the detection of the spatiotemporal (or movement) features of a stimulus, rather than by tasks involving active vs. passive touch as suggested by Wall (1970) and by Schwartzman and Semmes (1971).

\section{REFERENCES}

Azulay, A., \& Schwartz, A. S. The role of the dorsal funiculus of the primate in tactile discrimination. Experimental Neurology, 1975, 46, 315-332.

Gibson, J. J. Observations on active touch. Psychological Review, 1962, 69, 477-491. 
Schwartz, A. S., Eidelberg, E., Marchok, P., \& Azulay, A. Tactile discrimination in the monkey after section of the dorsal funiculus and lateral lemniscus. Experimental Neurology, $1972,37,582-596$.

Schwartzman, R. J., \& Semmes, J. The sensory cortex and tactile sensitivity. Experimental Neurology, 1971, 33, 147-158.

Semmes, J. Protopathic and epicritic sensation: A reappraisal. In A L Benton (Ed) Contributions to clinical neuropsychology. Chicago: Aldine, 1969. Pp. 142-171.

Vierck, C. J. Alteration of spatio-tactile discrimination after lesions of primate spinal cord. Brain Research, 1973, 58, 69-79.
Vierck, C. J. Tactile movement detection and discrimination following dorsal column lesions in monkeys. Experimental Brain Research, 1974, 20, 331-346.

Wall, P. D. The sensory and motor role of impulses travelling in the dorsal columns towards cerebral cortex. Brain, 1970, 93, 505-524.

Wartenberg, R. A "numeral test" in transverse lesions of the spinal cord. American Journal of Medical Science, 1939, 198 , 393-396.

(Received for publication March 3, 1975.) 